\title{
Cauda equina syndrome caused by primary and metastatic neoplasms
}

\author{
Carlos A. Bagley, M.D., AND ZiYa L. Gokaslan, M.D. \\ Department of Neurosurgery, The Johns Hopkins Hospital, Baltimore, Maryland
}

\begin{abstract}
Cauda equina syndrome (CES) is defined as the constellation of symptoms that includes low-back pain, sciatica, saddle anesthesia, decreased rectal tone and perineal reflexes, bowel and bladder dysfunction, and variable amounts of lower-extremity weakness. There are several causes of this syndrome including trauma, central disc protrusion, hemorrhage, and neoplastic invasion. In this manuscript the authors reviewed CES in the setting of both primary and secondary neoplasms. They examined the various primary tumor types in this region as well as those representative of metastatic spread. Both surgical and nonsurgical management in this setting were studied.
\end{abstract}

KEY WoRDS • cauda equina • neoplasm • surgery • metastasis

Cauda equina syndrome refers to a very specific constellation of symptoms that result from damage to the cauda equina, which refers to the portion of the nervous system below the conus medullaris and consists of peripheral nerves, both motor and sensory, within the spinal canal and thecal sac. Damage in this region causes a variety of symptoms, including sciatica; low-back pain; saddle and perianal hypesthesia or analgesia; decreased rectal tone; absent bulbocavernosus, patellar, and achilles reflexes; bowel and bladder dysfunction; and variable amounts of lower-extremity weakness. The causative agent may be extremely variable and includes lumbar disc herniations, arachnoiditis, hemorrhage, trauma, and neoplasms. In this manuscript we review CES in the setting of tumors. First, we look at the tumor types that are common to this region. Second, we review the relative incidence of metastatic tumors and the presentation of patients harboring these lesions as reported in the literature. Last, we examine outcomes following surgical and nonsurgical management of CES caused by neoplastic compression.

Patients with CES caused by a neoplasm often have a relatively long, nonspecific disease course. A long history of back pain and paresthesias as well as occasional urinary difficulties is very common. ${ }^{10}$ The patients with primary tumors are usually young and otherwise healthy, circumstances that often lead to a considerable delay between the onset of symptoms and final diagnosis. Patients with metastatic tumors are older and usually have a history of cancer. It is imperative that the condition of patients with a history of malignancy and new-onset back pain or without neurological deficit be taken seriously and that the appropriate imaging studies are obtained. In patients with no

Abbreviations used in this paper: $\mathrm{AP}=$ anteroposterior; $\mathrm{CES}=$ cauda equina syndrome; $\mathrm{CNS}=$ central nervous system; $\mathrm{CT}=\mathrm{com}-$ puterized tomography; $\mathrm{MR}=$ magnetic resonance. medical history of malignancy but in whom there is abnormal weight loss, hematuria, hemoptysis, melanotic stools, and so forth, the possible presence of a metastatic lesion should be suspected. Most investigations may begin with AP and lateral plain spine radiography studies. Findings that should prompt further investigation include pedicle or other bone erosion, abnormal calcifications, and new or worsening scoliosis in the setting of back pain. Patients demonstrating weakness or bowel and/or bladder dysfunction should undergo MR imaging studies with and without Gd enhancement of the appropriate spinal levels, as dictated by results of the clinical examination. Some patients may benefit from additional information gained through CT scanning. In addition to allowing superior assessment of bone integrity at the offending and adjacent levels, CT scanning allows one to differentiate between cauda equina compression from a soft-tissue mass and that from the bone elements, a distinction that may play a critical role in planning surgical rather than nonsurgical treatment.

\section{PRIMARY TUMORS}

\section{Myxopapillary Ependymoma}

In considering the various common tumor types that may cause CES, it is helpful to divide this spinal region into its various parts. First, there are tumors that arise from the conus medullaris or the terminal filum. The most common primary spinal cord tumor in this region $(83 \%)$ is the myxopapillary ependymoma. This subclass of ependymoma has a particularly high affinity for the terminal filum and is believed to arise from its ependymal glia. These tumors tend to occur in young adults with a mean age of 36.4 years at presentation. ${ }^{28,31,35}$ There is a $2.2: 1$ male/female ratio. These tumors tend to be very slow growing, and patients often present with a rather long history of 
back, leg, or sacral pain and weakness, or sphincter dysfunction.

Magnetic resonance imaging is the diagnostic modality of choice for visualizing the conus medullaris and cauda equina region. Myxopapillary ependymomas appear as well circumscribed, sausage-shaped lesions that are hypointense on $\mathrm{T}_{1}$-weighted sequences, hyperintense on $\mathrm{T}_{2}$-weighted sequences, and brightly enhanced with the addition of Gd. Microscopically, these lesions are characterized by cuboidal cells positive for glial fibrillary acidic protein and arranged in a rosette-like pattern. A mucoid matrix accumulates between the tumor cells and the vasculature, giving this tumor its distinct microscopic appearances (Fig. 1).27,35

Excision is the treatment of choice for myxopapillary ependymomas, with cure being possible on complete excision. Typically, there is a clear cleavage plane found intraoperatively because these tumors tend to compress adjacent neural tissue rather than invade it. In addition, the success of surgery has been shown to correlate directly with the degree of neurological deficit on presentation and inversely with the duration of symptoms ${ }^{18,30}$ Of those patients with only pain on presentation, $86 \%$ had excellent outcomes (that is, symptom free or minor symptoms not affecting daily living), whereas only $67 \%$ of those with motor weakness and 33\% of those with sphincter dysfunction on presentation had good outcomes. ${ }^{30}$

\section{Schwannomas of the Spinal Region}

Schwannomas are neoplasms arising from the nerve sheath. The transition zone from central myelin (oligodendrocytes) to peripheral myelin (Schwann cell) is known as the Obersteiner-Redlich zone, and this transitional region typically gives rise to schwannomas. ${ }^{5}$ These tumors may arise in a solitary, sporadic fashion (most common) or in multiples as part of a complex genetic disorder known as neurofibromatosis Type 2. Multiple schwannomas occasionally may occur in a sporadic fashion, but this is far less common. These tumors have a

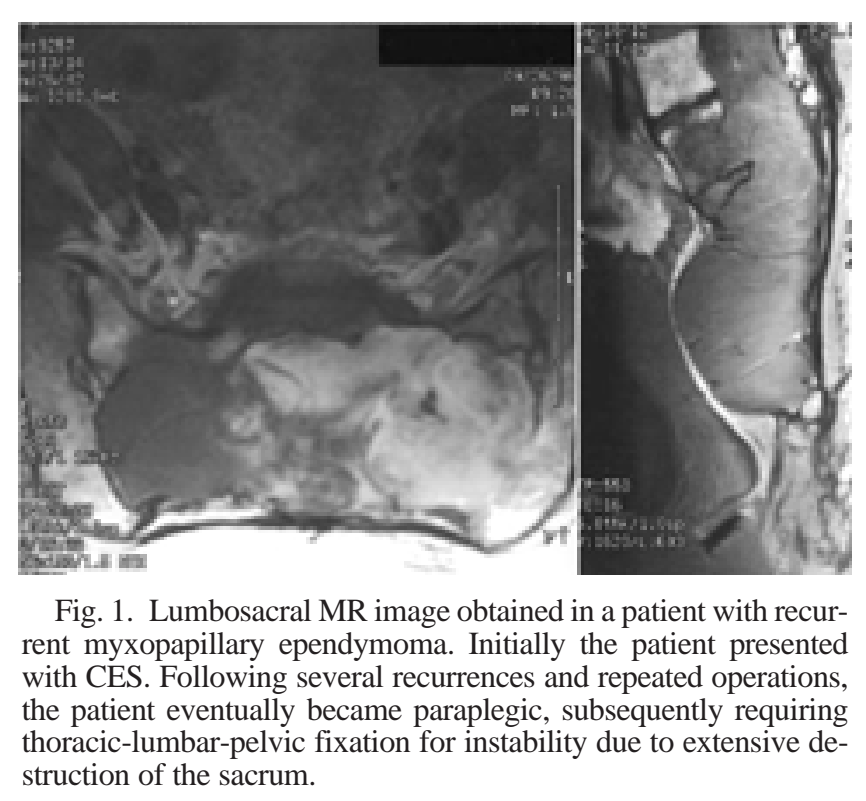

peak incidence in a patient's fifth and sixth decades. Furthermore, schwannomas have a predilection for sensory nerves and tend to arise from the dorsal roots.

On gross examination schwannomas are soft, discrete, lobulated masses that may or may not have a cystic component. Their color may vary from yellow to darkly pigmented in the melanotic variant. Small lesions may be seen as appendages from the nerve root of origin, whereas larger masses may dramatically deform and compress rather than invade neighboring roots. Microscopically, these tumors are characterized by spindle-shaped, neoplastic Schwann cells with alternating compact (Antoni type A) and loosely textured (Antoni type B) areas. ${ }^{35}$

Bone changes may be evident on plain radiographs in cases of schwannomas. Widening of the neural foramen and scalloping of the adjacent vertebral body are commonly visible. Computerized tomography scans may demonstrate associated osseous erosion along with a hypodense to slightly hyperdense mass. Calcifications may be seen rarely. Results of MR imaging studies are variable, although tumors most often appear isointense on $\mathrm{T}_{1^{-}}$ weighted sequences and hyperintense on $\mathrm{T}_{2}$-weighted sequences. ${ }^{28}$ This may vary depending on the fat and melanin content of some schwannoma subtypes. In addition, approximately $40 \%$ of schwannomas have a cystic component. Virtually all tumors, regardless of the subtype, enhance following administration of a contrast agent (Fig. 2). ${ }^{27}$

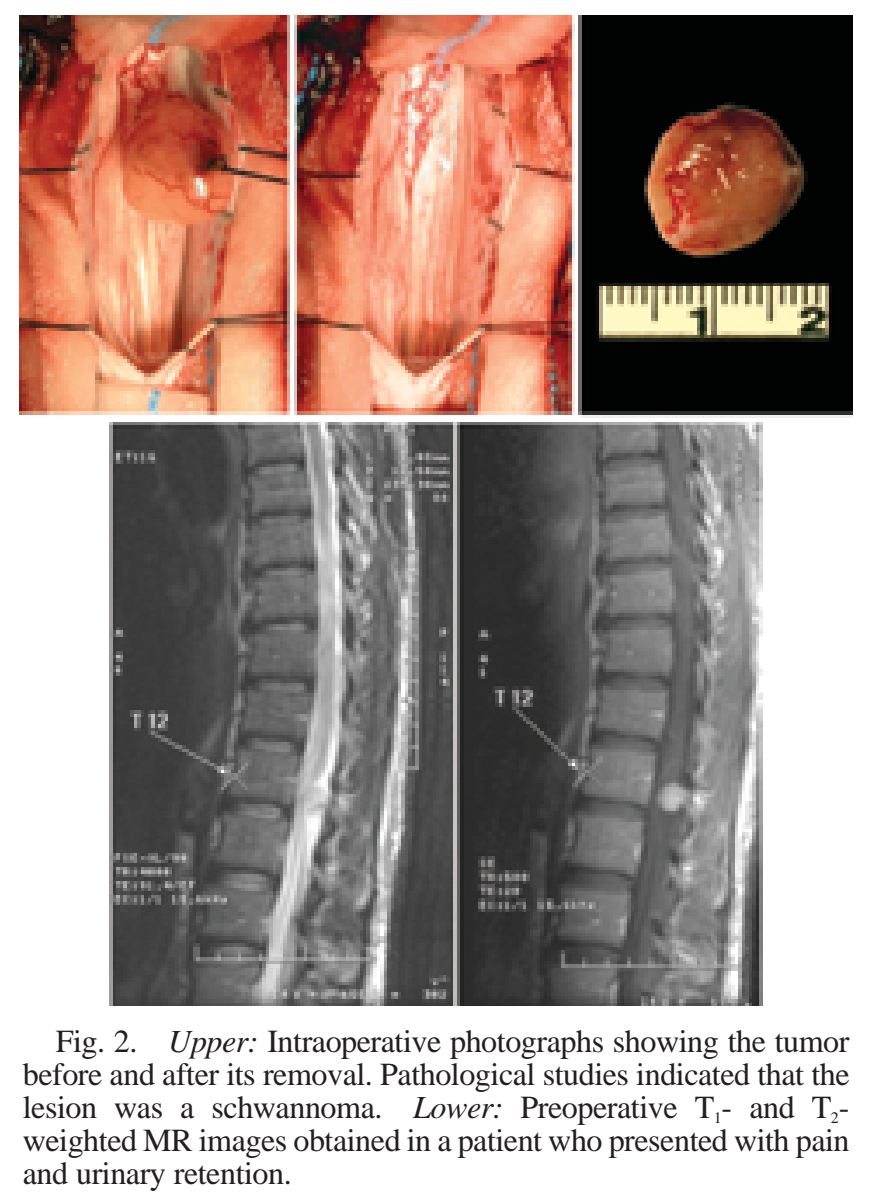


Clinical symptoms associated with spinal schwannomas are indistinguishable from those associated with disc herniation. ${ }^{27}$ Radicular pain is a common complaint, followed by paresthesias and weakness.

Excision is the treatment of choice for schwannomas. These tumors are very slow growing and well circumscribed and therefore are readily amenable to complete excision. No role has been defined for radio- or chemotherapy in the management of these benign lesions.

\section{Paragangliomas of the Spinal Region}

Paragangliomas are slow-growing, benign neoplasms of neuroendocrine origin and may rarely occur in the spinal cord. These tumors arise from the accessory organs of the peripheral nervous system known as the paraganglia. They may also be found in the adrenal gland (pheochromocytomas), the carotid body (carotid body tumors), or the immediate vicinity of the vagus nerve (vagus paragangliomas). The first description of a paraganglioma of the terminal filum was published in 1970 by Miller and Torack. ${ }^{28}$ Since the writing of this report, several other case reports and small case series involving paragangliomas of the spinal cord have been described, with more than 120 reported to date. Paragangliomas are usually intradural, extramedullary lesions with a high affinity for the cauda equina and terminal filum..$^{29,35}$ On gross examination, these are soft, encapsulated, homogeneous intradural masses

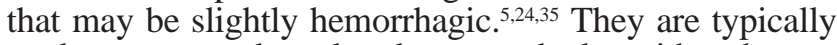
oval to sausage shaped and are attached to either the terminal filum or less often to a caudal nerve root. ${ }^{24,35} \mathrm{Mi}$ croscopically, paragangliomas are characterized by uniform cells with neuronal differentiation forming compact nests known as "zellballen" and are surrounded by a delicate capillary network (Fig. 3). ${ }^{35}$

As with other tumors of the spinal region, the most common symptoms on presentation are low-back pain and sciatica, with a mean symptom duration of 4 years. ${ }^{31} \mathrm{On}$ ly four endocrinologically functional (catecholamine-producing) paragangliomas have been reported to date. ${ }^{20,35}$ The mean age on presentation is in the fifth decade (that is, 47 years), with men being more often affected than women $(1.4: 1) .5,24,35$

Radiographically, cauda equina paragangliomas are fairly nonspecific. Findings on plain radiographs and CT scans include bone erosion (scalloping) and widened interpedicular distance. In addition, an isodense, homogeneously enhancing mass may be noted on CT scans. On $\mathrm{T}_{1}$-weighted MR images, these masses appear as isointense to slightly hypointense, with marked contrast enhancement. The $\mathrm{T}_{2}$-weighted image visualizes a hyperintense mass.

The prognosis for cauda equina paragangliomas is excellent. Given its encapsulated nature, complete excision affords a potential cure. The long-term recurrence rate following gross-total removal has been estimated at approximately $4 \% .{ }^{35}$ Seeding of the CNS has been reported, and there has been a single case report of metastasis outside the CNS. ${ }^{29}$ No clear role for radiotherapy in the treatment of residual or recurrent disease has been defined given the rare occurrence of this tumor and its high surgical cure rate.

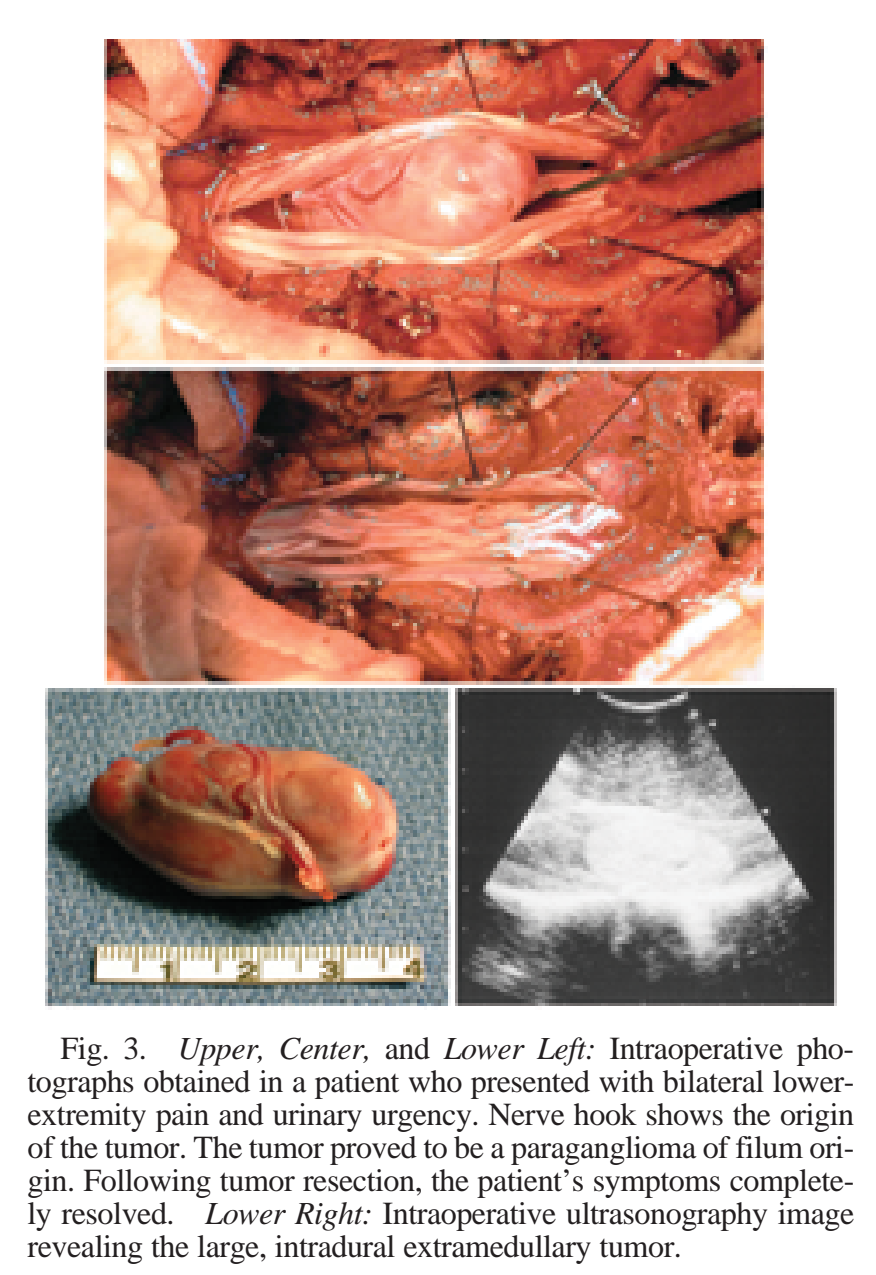

\section{Astrocytomas of the Spinal Region}

Astrocytomas are relatively common spinal cord tumors, especially in children, in whom it is the most common primary spinal cord lesion. ${ }^{24}$ These tumors are exceedingly rare in the region of the conus medullaris and terminal filum, however. Isolated conus medullaris involvement occurs in only approximately $3 \%$ of cases. ${ }^{24}$ These tumors, like other glial spinal neoplasms, tend to appear in patients who have a long history of back and leg pain along with variable amounts of motor weakness and sphincter dysfunction. Low-grade tumors (World Health Organization Grades I and II) often lack objective neurological signs in patients at presentation, thus often delaying diagnosis. On the other hand higher-grade tumors (World Health Organization Grades III and IV) tend to have a much shorter duration of symptoms and demonstrate significantly more neurological symptoms on presentation. ${ }^{9}$

On gross examination, astrocytomas appear as diffuse fusiform enlargements of the spinal cord or conus medullaris. These tumors are characterized by increased cellularity along with variable amounts of cellular pleomorphism, nuclear mitoses, and necrosis, depending on the tumor grade. In addition, these tumors lack a clear cleavage plane, and neoplastic cells tend to infiltrate well beyond the region of radiographic abnormality. ${ }^{24}$ On plain 
radiographs and $\mathrm{CT}$ scans, bone erosion and widened interpedicular distance may be seen. There may or may not be associated scoliosis and canal widening. Magnetic resonance imaging is the modality of choice in visualizing astrocytomas of this region. On $\mathrm{T}_{1}$-weighted images, the tumor tends to be isointense to hypointense to the normal spinal cord, with variable amounts of enhancement on the administration of a contrast agent. On $\mathrm{T}_{2}$-weighted images, the lesions appear hyperintense.

The prognosis for astrocytomas of the conus medullaris and terminal filum is far worse than that for ependymomas. Grade I lesions, which occur mainly in children, may possess a cleavage plane, and therefore gross-total excision may be possible. In contrast, higher-grade lesions, which occur predominantly in adults, lack a clear cleavage plane and have a relatively higher recurrence rate. Furthermore, unlike with ependymomas, the extent of resection in astrocytomas does not affect overall patient survival because the lesions are infiltrative with a high recurrence rate. ${ }^{7}$ This is one clinical situation in which a frozen-section diagnosis determined intraoperatively is critically important. Should the characteristics of the frozen section be consistent with those of an infiltrating glioma and no clear cleavage plane is found, then tumor debulking should be undertaken with the intent of minimizing trauma to functional tissue. In this circumstance, postoperative radiotherapy should be administered in an attempt to control residual disease..$^{25}$

\section{Chordomas of the Spinal Region}

Chordomas are malignant tumors that arise from the remnants of the primitive notochord. In the spine, the most common location for this slow-growing, aggressive tumor is the sacrum and the coccyx. Chordomas account for 2 to $4 \%$ of malignant primary osseous neoplasms and are the most common primary sacral neoplasm. ${ }^{8,27}$ The peak incidence for sacral chordomas is the sixth decade, and there is a 2:1 male predominance. ${ }^{8}$

Sacral chordomas are locally invasive, lobulated masses with a gelatinous texture. Two subsets of this tumor exist: typical chordomas and chondroid chordomas. In the typical type, variable amounts of intracellular mucin are embedded in pools of extracellular mucin. In the chondroid variant this gelatinous mucinous matrix is replaced by cartilaginous foci. Microscopically, the hallmark of chordomas is the vacuolated, bubble-bearing cell know as "physaliferous cell."

Neuroimaging findings are extremely variable when dealing with chordomas. On $\mathrm{T}_{1}$-weighted $\mathrm{MR}$ imaging sequences there tends to be a predominantly low signal, whereas on $\mathrm{T}_{2}$-weighted and proton-density images the signal is predominantly equal to or greater than that of cerebrospinal fluid. The enhancement pattern varies from very little to intense. Often there are solid and cystic components. Calcifications may be visualized on CT scans, and often an associated anterior or lateral soft-tissue mass is demonstrated as well. The typical CT scan demonstrates a mixed lytic and sclerotic picture. ${ }^{30}$ In addition, these tumors are capable of extending across the disc space and the sacroiliac joint. Plain radiographs often reveal significant sacral erosion and enlargement of the AP diameter of the sacrum (Fig. 4).
The most common presenting symptom in a patient harboring a sacrococcygeal chordoma is pain. Usually it confined to the low back, but sciatic-type pain has also been reported in up to $30 \%$ of patients. ${ }^{13,21}$ Furthermore, patients have a high incidence of constipation, likely due to the enlarging presacral mass that causes rectal compression. The mean duration of symptoms by the time of patient presentation ranges from 1 to 3 years because of the slow rate of the tumor's growth. ${ }^{30}$

Resection is the treatment of choice for sacral chordomas. Histologically, chordomas are relatively low-grade lesions. Nonetheless, they tend to develop in locations such as the clivus and the sacrum, which makes surgical cure extremely difficult. Note that gross-total resection has been demonstrated directly to affect long-term survival. ${ }^{21,36}$ The role of radiation treatment in the overall management of sacral chordomas is rather controversial. Traditionally, $\mathrm{x}$-ray beam radiotherapy has been shown to be marginally effective to completely ineffective in the treatment of these lesions. There is, however, an emerging body of data that supports the use of proton-beam radiotherapy for lesions not amenable to resection and for residual/recurrent disease. Chemotherapy has been shown to have no value in the treatment of chordomas.

\section{Giant Cell Tumors of the Spine}

Giant cell tumors are locally aggressive, primary bone tumors that typically occur at the ends of long bones. Although only approximately $7 \%$ of giant cell tumors occur in the spine, the most common spinal location is the sacrum. This lesion is the second most common primary sacral tumor after chordomas. ${ }^{8}$ These tumors represent approximately $5 \%$ of all primary bone tumors and have a peak incidence in the third decade of a patient's life. There is a slight female predominance for giant cell tumors involving the spine. $8,27,35$

Giant cell tumors are locally aggressive and destroy local bone. Typically they progress through the sacral cortex, but rarely through the periosteum. Histologically, they contain sinusoidal vessels with hypervascular stroma. The giant cells, which are multinucleated macrophages, are not specific to giant cell tumors and may occur as a reaction to foreign tissue. ${ }^{8}$ Although typically histologically benign, approximately 5 to $10 \%$ of giant cell tumors may undergo malignant degeneration and assume a more aggressive course.

Common findings on plain radiographs of giant cell tumors include erosion of the sacral bone, widening of the sacral canal, and widened interpedicular distances. On CT scans, giant cell tumors appear as soft-tissue masses that may or may not possess a sclerotic rim. These lesions are very vascular and demonstrate intermediate signal intensity on both $\mathrm{T}_{1}$ - and $\mathrm{T}_{2}$-weighted MR imaging sequences. Often there is intense contrast enhancement with the administration of Gd. Angiography studies may also demonstrate the intense vascularity of these tumors and may be instrumental in preoperative embolization for planned surgical intervention.

Excision is the treatment of choice in giant cell tumors of the sacrum. Given the large size and the destructive nature of these lesions, surgical management may be very complex and necessitate a multidisciplinary approach 


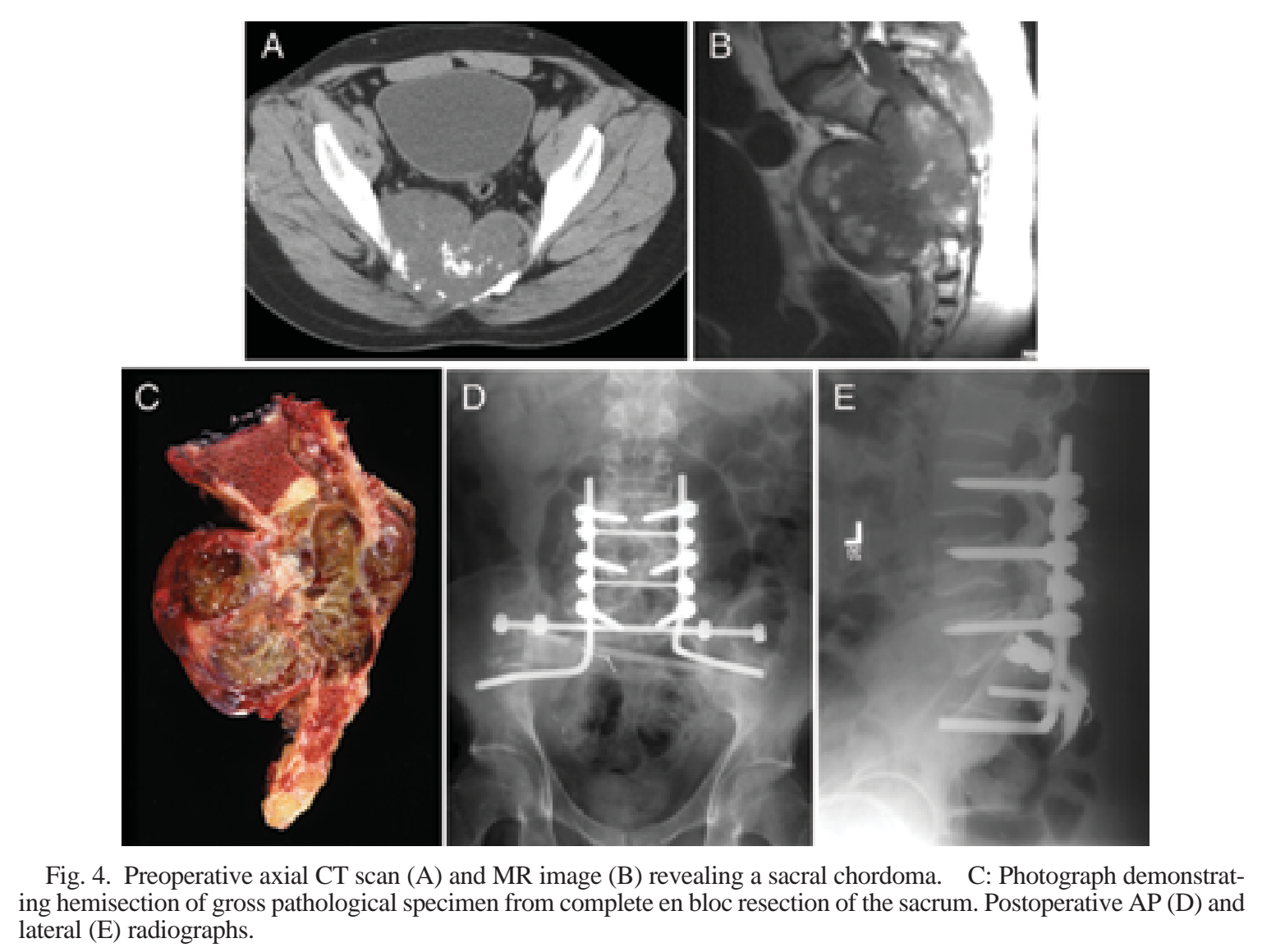

both to excise and reconstruct this region. Local recurrence following excision is fairly common and occurs in approximately $20 \%$ of cases. ${ }^{15}$ In addition, malignant transformation is a serious concern with giant cell tumors, occurring in up to $10 \%$ of cases. ${ }^{8,27}$ For recurrent or residual disease, radiation may be a useful adjunctive treatment. ${ }^{6,23}$ Several groups have been able to demonstrate reasonable tumor control in subtotally resected lesions involving the sacrum. ${ }^{6,23}$ There is no clear role for chemotherapy in the management of giant cells tumors of the spine.

\section{METASTATIC TUMORS}

Metastatic tumors comprise the bulk of all tumors occurring in the spine in general. Note, however, that isolated metastases to the low lumbosacral region or the cauda equina are relatively rare compared with the primary tumors occurring in this region. With the improved treatments of many systemic cancers and the resultant increase in patient survival, spinal metastases and their sequelae are becoming increasingly common. Of all primary lesion types, lung carcinoma (40-85\%), breast carcinoma (11\%), renal cell carcinoma (4\%), lymphoma (3\%), and colorectal carcinoma (3\%) are the most common types seen in the spine. These tumors may primarily involve bone or may be intradural or extradural in location. Intramedullary metastases are extremely rare, especially in the region of the conus medullaris. Intracranial lesions such as ependymo- mas, primitive neuroectodermal tumors, and glioblastomas multiforme may have drop metastases to the lumbosacral region. Spread to the lumbosacral spine may also occur by direct invasion, as in the case of some gastrointestinal and genitourinary cancers, or by hematogenous spread, which is the most common route. Signs and symptoms of neural involvement may be caused by direct tumor invasion or from bone compression due to pathological spine fractures.

The radiographic findings in the setting of metastases are for the most part nonspecific. The presence of multiple lesions involving the lumbosacral region is, however, an imaging hallmark of metastases. Metastatic lesions are usually osteolytic, although breast and prostate cancer may appear as sclerotic masses. ${ }^{8,27}$ Plain radiographs may demonstrate lytic or sclerotic regions. Computerized tomography scanning may further help to delineate the mass as well as assess the bone quality of the affected and adjacent levels. Magnetic resonance imaging studies again demonstrate multiple lesions whose signal intensities vary depending on the primary lesion. In cases in which drop metastases are suspected, it is imperative to obtain an image of the brain as well as the spine; this may prevent potentially disastrous tonsillar herniation from a large posterior fossa mass.

In general, patients with epidural metastatic spinal lesions localized to the cauda equina region present with symptoms similar to those in patients with primary tumors. Low-back pain, numbness, paresthesias, and vari- 
able amounts of sphincter dysfunction are common. Symptoms of severe pain preceding the onset of motor weakness generally correlates with epidural compression, whereas the appearance of muscle weakness and sphincter dysfunction with very little pain generally indicates intramedullary metastasis. ${ }^{11,14,26}$

As mentioned earlier, lung cancer is the most common tumor to metastasize to the spine. Tumors usually spread to the thoracic region, and compression at the level of the cauda equina is relatively rare. In a large retrospective review of more than 2200 patients with lung cancer from two large university centers, Ampil, et al., ${ }^{3}$ found only 16 cases $(0.7 \%)$ of cauda equina compression from metastatic lung cancer. In this review, there was a long duration of symptoms (mean 89 days) prior to diagnosis. The most common subtype of metastatic lung cancer found in this review was non-small cell. Most patients with cauda equina compression from metastatic lung cancer were also found to have metastases elsewhere in the body at the time their disease was diagnosed. The overall prognosis for the disease in this group of patients is extremely dismal, with a mean survival of only 2 months. This is consistent with the overall prognosis in patients with metastatic lung cancer at other vertebral levels in whom mean survival has been found to be approximately 3 months. ${ }^{33,34}$

Breast cancer represents the second most common origin of symptomatic spinal metastases. Approximately $80 \%$ of patients with breast cancer will have skeletal metastases during the course of their disease. ${ }^{4}$ Patients with breast cancer may present with diffuse metastatic meningeal carcinomatosis in the cauda equina region, similar to lung cancer. In one large retrospective study (1283 patients), the incidence of cauda equina compression from breast cancer metastases was found to be $1.2 \% .^{1}$ The median survival in these patients was approximately 8 months (Fig. 5).
Lymphoma in most cases involves the epidural compartment and bone. Approximately 3\% of CNS lymphomas have intramedullary involvement, however. ${ }^{24}$ Intramedullary CNS lymphoma in the region of the conus medullaris or cauda equina is exceedingly rare and will not be discussed further in this paper. The far more common site of lymphoma involvement in this region involves infiltration of the bone elements, especially the sacrum.

Some metastatic tumors, such as thyroid or renal cell carcinoma, may be extremely vascular. Preoperative angiography studies and embolization are vital in these cases to prevent massive intraoperative blood loss. Definitive surgery is usually undertaken within 24 hours of the embolization procedure to minimize revascularization of the lesion.

\section{TREATMENT OPTIONS}

The clinical dogma that surrounds CES supports emergent decompression in patients presenting with the clinical hallmarks. A critical review of the literature, however, demonstrates that this issue is far more controversial than one might imagine. Outcomes in patients with CES vary according to the study one considers. Very few studies have been focused on outcomes solely in the face of neoplastic compression. Most treatment guidelines have therefore been extrapolated from the literature on CES in the setting of large disc herniations. As with spinal cord compression in other regions, outcomes appear to be far more favorable in patients with incomplete lesions compared with those harboring complete lesions. The outcomes in patients who undergo surgical decompression are very controversial. Some researchers have failed to find any difference between patients who undergo surgery emergently $(<12$ hours) and those who undergo surgery urgently (24-48 hours). ${ }^{19}$ Others, however, have found that those patients with incomplete lesions (that is, those

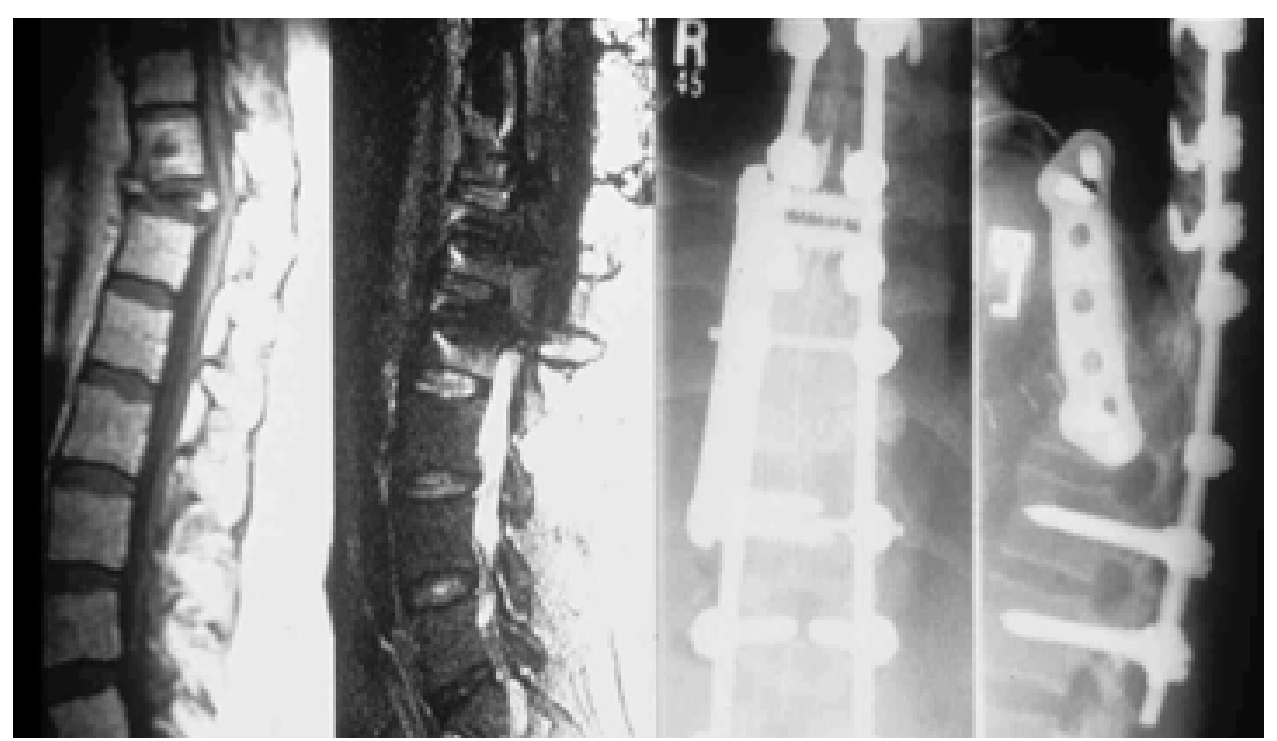

Fig. 5. Plain AP and lateral x-ray films and preoperative MR images obtained in a patient with known breast cancer who presented with CES. Postoperative lateral and AP x-ray films show final reconstruction. The patient first underwent anterior approach, T-11 and T-12 corpectomy, polymethylmethacrylate reconstruction, and plate fixation. During the second stage, the patient had supplemental posterior thoracolumbar fixation and fusion. The patient made a complete recovery. 
with neurogenic urinary difficulties, loss of the urge to void, and the need to strain to micturate) fared far better with emergent surgical decompression than did those with complete lesions (those with painless urinary retention and overflow incontinence)..$^{12,22}$

Analysis of data from animal models demonstrates that there is a very narrow window of opportunity during which surgical decompression may improve outcomes. ${ }^{22}$ Stephenson, et al., ${ }^{32}$ reported data from an experimental model of cauda equina compression in macaque monkeys. They found that decompression performed within 1 hour of syndrome onset had excellent outcomes. Furthermore, they noted that the benefit of surgical decompression had waned by 4 hours after syndrome onset. As one might imagine, this poses a great challenge in a clinical setting because the actual time of syndrome onset is often difficult to ascertain given that patients commonly have a fairly long history of chronic back pain and most often present after experiencing an inability to void or perianal anesthesia. These symptoms may occur hours after the actual onset of nerve root compression.

In addition, reported outcomes in the literature vary according to the outcome measure used. Those studies in which patients' subjective symptoms are used tend to be associated with much higher success rates on surgical decompression compared with the rates in urodynamic studies. Urodynamic studies may demonstrate significant bladder dysfunction despite a complete absence of patient complaints. ${ }^{16}$

Radiotherapy may be considered an option in patients with a fairly short life expectancy, in those who may not tolerate a trip to the operating room because of medical comorbidities or systemic disease, and in those whose compression is caused by a radiosensitive primary or secondary tumor. One must be aware that in cases of pathological fractures with bone fragments retropulsed into the spinal canal, radiotherapy will not be as effective as it might be in a scenario involving compression from the soft-tissue mass. Ampil, et al., ${ }^{2}$ reported their 19-year experience with palliative radiotherapy in patients with metastatic lesions and CES. Although this study was retrospective and had a relatively small sample size (76 patients), the authors did report excellent results in terms of pain relief (94\% complete relief and 3\% partial relief). These authors found similarly excellent results in regard to pain relief in patients with metastatic lung cancer (five of five patients improved significantly). ${ }^{3}$ Nonetheless they also found that very few patients experienced any improvement in impaired sensation or sphincter dysfunction compared with their pretreatment condition. Conclusions regarding motor strength improvement or worsening were not reported in this paper. These authors did, however, report that 11 of the 28 patients with some pretreatment "motion impairment" were ambulatory at the conclusion of treatment. This indicates that in the subgroup of patients with a fairly short life expectancy, palliative radiotherapy may be considered as a treatment alternative sufficient to improve their quality of life.

\section{CONCLUSIONS}

Cauda equina syndrome caused by neoplastic compression can have a very profound effect on the quality of life and overall survival of patients with cancer. A myriad of both primary and secondary (metastatic) lesions may occur in this fashion. In general, prompt diagnosis and treatment are the most important goals, because the timing of intervention may significantly affect the overall disease prognosis in these patients. Last, a multidisciplinary team that includes neuroradiologists, neuropathologists, radiation oncologists, and neurosurgeons is essential for the optimal management of this complex and potentially devastating condition.

\section{References}

1. Ampil FL, Burton GV, Mills GM, et al: Cauda equina compression in breast cancer-incidence and treatment outcome. Eur J Gynaecol Oncol 22:257-259, 2001

2. Ampil FL, Caldito G, Heldmann M: Palliation and survival in metastatic tumors compressing the cauda equina: a 19-year radiotherapeutic experience. J Palliat Med 6:33-36, 2003

3. Ampil FL, Mills GM, Burton GV: A retrospective study of metastatic lung cancer compression of the cauda equina. Chest 120:1754-1755, 2001

4. Bendre M, Gaddy D, Nicholas RW, et al: Breast cancer metastasis to bone: it is not all about PTHrP. Clin Orthop 415 (Suppl):S39-S45, 2003

5. Burger PC, Scheithauer BW, Vogel FS: Surgical Pathology of the Nervous System and Its Coverings, ed 4. Philadelphia: Churchill Livingstone, 2002, pp 540-543

6. Caudell JJ, Ballo MT, Zagars GK, et al: Radiotherapy in the management of giant cell tumor of bone. Int J Radiat Oncol Biol Phys 57:158-165, 2003

7. Cooper PR: Outcome after operative treatment of intramedullary spinal cord tumors in adults: intermediate and longterm results in 51 patients. Neurosurgery 25:855-859, 1989

8. Diel J, Ortiz O, Losada RA, et al: The sacrum: pathologic spectrum, multimodality imaging, and subspecialty approach. Radiographics 21:83-104, 2001

9. Epstein FJ, Farmer JP, Freed D: Adult intramedullary astrocytomas of the spinal cord. J Neurosurg 77:355-359, 1992

10. Fearnside MR, Adams CB: Tumours of the cauda equina. $\mathbf{J}$ Neurol Neurosurg Psychiatry 41:24-31, 1978

11. Gilbert H, Apuzzo M, Marshall L, et al: Neoplastic epidural spinal cord compression. A current perspective. JAMA 240: 2771-2773, 1978

12. Gleave JR, Macfarlane R: Cauda equina syndrome: what is the relationship between timing of surgery and outcome? $\mathbf{B r} \mathbf{J}$ Neurosurg 16:325-328, 2002

13. Gray SW, Singhabhandhu B, Smith RA, et al: Sacrococcygeal chordoma: report of a case and review of the literature. Surgery 78:573-582, 1975

14. Grem JL, Burgess J, Trump DL: Clinical features and natural history of intramedullary spinal cord metastasis. Cancer 56: 2305-2314, 1985

15. Hart RA, Boriani S, Biagini R, et al: A system for surgical staging and management of spine tumors. A clinical outcome study of giant cell tumors of the spine. Spine 22:1773-1783, 1997

16. Hellstrom P, Kortelainen P, Kontturi M: Late urodynamic findings after surgery for cauda equina syndrome caused by a prolapsed lumbar intervertebral disk. J Urology 135:308-312, 1986

17. HogenEsch RI, Staal MJ: Tumors of the cauda equina: the importance of an early diagnosis. Clin Neurol Neurosurg 90: 343-348, 1988

18. Hoshimaru M, Koyama T, Hashimoto N, et al: Results of microsurgical treatment for intramedullary spinal cord ependymomas: analysis of 36 cases. Neurosurgery 44:264-269, 1999

19. Hussain SA, Gullan RW, Chitnavis BP: Cauda equina syndrome: outcome and implications for management. Br J Neurosurg 17:164-167, 2003 


\section{A. Bagley and Z. L. Gokaslan}

20. Jeffs GJ, Lee GY, Wong GT: Functioning paraganglioma of the thoracic spine: case report. Neurosurgery 53:992-995, 2003

21. Kalmon DP, McCormick PC: Surgical management of pelvic tumors with intraspinal extension, in Schmidek $\mathrm{HH}$ (ed): Schmidek \& Sweet Operative Neurosurgical Techniques: Indications, Methods and Results, ed 4. Philadelphia: WB Saunders, 2000, pp 2303-2321

22. Kennedy JG, Soffe KE, McGrath A, et al: Predictors of outcome in cauda equina syndrome. Eur Spine J 8:317-322, 1999

23. Khan DC, Malhotra S, Stevens RE, et al: Radiotherapy for the treatment of giant cell tumors of the spine: a report of six cases and review of the literature. Cancer Invest 17:110-113, 1999

24. Koeller KK, Rosenblum RS, Morrison AL: Neoplasms of the spinal cord and filum terminale: radiologic-pathologic correlation. Radiographics 20:1721-1749, 2000

25. Lindstadt DE, Wara WM, Leibel SA, et al: Postoperative radiotherapy of primary spinal cord tumors. Int J Radiat Oncol Biol Phys 16:1397-1403, 1989

26. Orendacova J, Cizkova D, Kafka J, et al: Cauda equina syndrome. Prog Neurobiol 64:613-637, 2001

27. Osborn AG: Diagnostic Neuroradiology. St Louis: Mosby, 1994, pp 906-910

28. Miller CA, Torack RM: Secretory ependymoma of the filum terminale. Acta Neuropathol 15:240-250, 1970

29. Moran CA, Rush W, Mena H: Primary spinal paragangliomas: a clinicopathological and immunohistochemical study of 30 cases. Histopathology 31:167-173, 1997

30. Schweitzer JS, Batzdorf U: Ependymoma of the cauda equina region: diagnosis, treatment, and outcome in 15 patients. Neurosurgery 30:202-207, 1992
31. Sonneland PR, Scheithauer BW, LeChago J, et al: Paraganglioma of the cauda equina region. Clinicopathologic study of 31 cases with special reference to immunocytology and ultrastructure. Cancer 58:1720-1735, 1986

32. Stephenson GC, Gibson RM, Sontag VKH: Who is to blame for the morbidity of acute cauda equina compression? J Neurol Neurosurg Psychiatry 57:388, 1994 (Abstract)

33. Tatsui H, Onomura T, Morishita S, et al: Survival rates of patients with metastatic spinal cancer after scintigraphic detection of abnormal radioactive accumulation. Spine 21:2143-2148, 1996

34. Wedin R, Bauer HC, Rutqvist LE: Surgical treatment for skeletal breast cancer metastasis: a population-based study of 641 patients. Cancer 92:257-262, 2001

35. Wiestler OD, Schiffer D, Coons SW, et al: Myxopapillary ependymoma, in Kleihues P, Cavenee WK (eds): Pathology and Genetics of Tumours of the Nervous System. Lyon: IARC Press, 2000, pp78-79

36. York JE, Kaczaraj A, Abi-Said D, et al: Sacral chordoma: 40year experience at a major cancer center. Neurosurgery 44: 74-80, 1999

Manuscript received April 26, 2004.

Accepted in final form May 21, 2004.

Address reprint requests to: Ziya L. Gokaslan, M.D., Department of Neurosurgery, Meyer Building 7-109, The Johns Hopkins Hospital, 600 North Wolfe Street, Baltimore, Maryland 21287-7713. email: zgokas11@jhmi.edu. 